\title{
Design and Implementation of Educational Application for Directorate of Traffic Safety, Oman
}

\author{
Halima AL Hamdania \\ Noof Al Saadi \\ Eqtibas Al-Moqbali \\ Vikas Rao Naidu \\ Raza Hasan
}

\author{
Middle East College \\ Middle East College \\ Middle East College \\ Middle East College \\ Middle East College
}

\begin{abstract}
The world always faces multiple risk factors due to an increase in traffic accidents. This leads to the death of many people. Some of them get seriously injured and get permanent disabilities due to the negligence of persons in following traffic safety procedures. The General Directorate of Traffic in the Sultanate of Oman is responsible for regulating traffic in the country by monitoring the movement of traffic in the streets of inside cities and outside to preserve the safety of traffic in the streets of the Sultanate of Oman. This research paper aims to provide a solution in educating Omani residents about teaching driving laws, especially in Oman. The solution will raise awareness in the society with the rules and laws related to traffic safety. In order to justify and support the requirements of this research, the researchers conducted interviews and surveys among the public. The methods of data collection included questionnaires and interviews.

The analysis showed that majority of the audience who participated in surveys strongly agreed that such kind of education system for the residents was really essential so that they could learn about the importance of traffic rules and regulations right from their childhood. Finally, the researchers have proposed to develop a mobile app, which would be one of its kind and exclusive in Oman by Royal Oman Police (ROP) - Traffic Safety Unit. The methodology used in the development of this project would be the spiral model. Relevant literature was reviewed in order to study the existing system and also the similar systems which are existing around the world.
\end{abstract}

\section{Introduction}

General Directorate of Traffic is the organization which is responsible for organizing traffic in the Sultanate of Oman. The directorate ensures safety through monitoring the movement of traffic in the streets inside cities and outside. It helps to maintain the safety of traffic in the streets of the Sultanate of Oman through the issuance of laws and procedures that help in the deployment and preservation of traffic safety in the streets of the Sultanate of Oman. It also maintains patrols in routine street surveillance and uses the monitoring and communication devices. They target at reducing accidents as well as regulating traffic. The Directorate General of Traffic is not limited to organizing traffic. Among its other works are transfer of injured from accident locations to the nearest hospital, investigating the incident, issuing the license of the driver, registering and inspecting vehicle validity and determining the damages of traffic accidents, and, disseminating the laws of traffic safety through its awareness campaigns. (traffic.gov,2018)

The search paper talks about creating an App about teaching driving laws. It is an educational App. In the field of smart education, it explains the traffic safety rules that must be followed during learning the driving in accordance with the standards of traffic safety at the Directorate General of Traffic in the Sultanate of Oman. The beneficiary of this App is the Institute of Traffic Safety and 


\section{Journal of Student Research}

Fourth Middle East College Student Research Conference, Muscat, Sultanate of Oman

the General Directorate of Traffic in addition to the trained and people who need to learn driving. As the system of learning driving changes and requires the person to enter the Institute for study before entering for practical driving tests, the App design is practical and useful to all. In addition to enjoying learning the laws, the App makes it easier for one to memorize and learn the rules and regulations. Unlike traditional methods, the App includes laws and procedures that must be followed in order to obtain a license from the beginning to the end, tests, and the new laws issued by the General Administration of Traffic.

Objectives of literature review when writing during the start of the project. There are four main objectives. Search for literature in the field of study, collect and summarize the information contained in the literature. By analyzing the information gathered. Show the views of my topic in addition to the answers and negatives of the subject. The final Objective is writing it in an orderly manner in terms sequence of information.

The user interface is an application that allows the persons to communicate with the machines through it and controls the system and interacts with it. Also, the other meaning for user interfaces is the face of the application used for interacting with humans. There are different types of the user interfaces such as graphical user interface, web based-user interface, command-line interface, tactile and touch interface. This article is about the measuring satisfaction of users who use interface of the gaming in Smartphones. The objective of this research is to satisfy the users of the games by designing the interface. And use user-friendly themes in in-game interfaces. Easy and simple design to users use. In this study, five of the most famous games were chosen in the past years. And study the Interface the game and reactions of users. In this time the designers care a lot about users' opinions. Because there are many competing Games in designing games world.

Participated in this study the players of the games and conducted four tests for them. To take their opinion about two surveys the comfort when using the system and user's satisfaction. The results of the survey showed that players prefer to use easy-to-control system interfaces, clear and simple icons, simple updates and instructions for use. In the end, this article to get Satisfied Users Phone Games. This article is useful in the project. useful in the design interface of the project. Important to understand the user's requirement in interface design App in the project. In designing the App must be attention to seven things. Who are peoples use the system, simplicity of design, consistency in design, pattern usage, rendering of notes and use of shortcuts in the system. (Hsu,2018).

3D graphics is using modern technology. Most famous programs draw three - dimensional 3Ds Max, Maya and Blender. The three-dimensional drawing is used for purposes such as filmmaking, games, and architectural engineers. 3Ds Max is a program to draw three-dimensional graphics for movies and games. From Autodesk company. This book about the design of bend muscle speed in the 3Ds Max software. The book supports pictured educational programs step by step. There are examples of the transition from start to finish and save objects with the project. The book explains the design of the methodological assets beloved. In the end, this book is important for anyone who wants to develop his skills in 3D design, game developers and filmmakers. It's useful for the project. To improve skills in design 3D. (Mooney, Thomas, 2012)

Unity is a Software used to create two - and three - dimensional games. The software works on many platforms such as Windows and Linux. And in mobile phones such as ISO and ...etc. Languages used in the software C\#, JavaScript and Boo. Used to the programming of games. This article about 3D Modeling in Unity software and its features and challenges. The use of 3D models in city management has become commonplace these days. Where used in the drawing of buildings and bridges and others. It has been used in many research and implementation projects. In order to see the projects more realistically. Like, Use a three-dimensional model in the potential solar energy project of BIMTAS company in Istanbul. In this paper search for 3D and modelling in 3D. Project implementation and phases and steps of the project. In addition, the advantages and limitations of using the Unity software. At the end of this article discussed the possibility of using solar energy and noise on buildings, bridges, underground facilities and other places. The 


\section{Journal of Student Research}

Fourth Middle East College Student Research Conference, Muscat, Sultanate of Oman

advantage of Unity is easy to use, available on the market and convenience on many platforms such as windows and mobile phones. Its disadvantages require improved perception, the environment, its database and other functions. This article is important and useful in the project. To know the steps of establishing the project on the software of Unity and its negative aspects and advantages. How are the stages of design and modelling in the software. (Buyuksalih, Ismail; Bayburt, Serdar; Buyuksalih, Gurcan; Baskaraca, A. P.; Karim, Hairi; Rahman, Alias Abdul, 2017)

This article about learns the advantages of advanced knowledge in 3D modelling in the Unity software. One of the objectives of the studies presented in this article about knowledge and learning in the advantages of 3D modelling. The study focused on learning to $3 \mathrm{~d}$ design in space, moving, scaling and the ability to balance and use the skills. and realizing the measurement of virtual areas of 3D and measurements. Whereas the creation of a project in the Unity software requests a suitable user interface with include both visualize the 3D environment and adapt to suit the advantages of the project. The researcher used in the research methods of this study such as research in literature and lessons he collected from his students and observations and assessments of the situation. How important it is to know the effects of 3Ds Max software in learning and design in unity software. Since one of the most important results reached by the researcher after the completion of the research is the prior knowledge in three-dimensional software. And the use of its effect is important to the designer and programmer before starting to use the Unity software is very important. Such as 3Ds Max, Maya, and Blender. This makes it easier to work on the Unity software for both the designer and the programmer. Therefore, it is important before to start the design of the project to have a background in one of this software. (Serdar,2019)

This book about design games. Talks about design the game in unity step by step. From start step to end step. The book contains integrated examples that help to create the game fully. And exercises and external references to develop the level of game design and designer skills. This book is useful for game designers, designers and 3D engineers who want to create interaction with the design. With little use of scripting language or without them. This book is more specialized in people who want to create a game from the start in the Unite software or Playmaker software. At the end of this book is very important to have a background on programming and about the Unity software. To start designing the 3d Game in Unity. This book is important and useful in proportion to the design of the game step by step. There are many examples, exercises and external resources that develop my skills in design and game level. (Mohov, Sergey, 2013)

This article about traffic safety and the beliefs of Oman police in traffic in Oman. In recent years, the proportion of vehicles in the Sultanate of Oman has increased. Resulting in an increased risk of roads. One of the functions of the Oman Police maintain traffic and organize roads throughout Oman. Which helps to improve the movement of traffic on the roads. As for the General Directorate of Traffic, it is responsible for the control of traffic laws. In this study, IS conducted with nineteen police officers in different areas of the Oman police. The results of this study most commonly define the importance of the role of the police in maintaining traffic safety. This is due to a positive side to road safety in Oman. In proportion to a few of them do not know the importance of the presence of the police. And their role in maintaining safety in the roads. At the end of this article, the study identified other areas to focus on improving the functioning of the Omani police. The functional objective of this work.

And may affect the work environment. This article is important in the work of the Omani police in maintaining peace and moving the roads. For application in project creation. And what are the views of the police and what are the important factors when designing the application. (Al Mazruii, Mudhar, Edwards, Jason, Armstrong, Kerry, Davey, Jeremy,2015)

This article about SDLC to develop the software. SDLC is the Software Development Life cycle. It is used for engineering systems, information systems and software engineering. It is a software development process in software. It consists of separate stages in the development, maintenance and replacement of specific programs. The article contains a sequence of stages in software 


\section{Journal of Student Research}

engineering. To be included in the proposed program. SDLC is used after completing the project planning stage. Which helps to develop projects. This study discussed an important subject in the computer world. This study includes the management of programs in software development through the use of development models. And programs used in software development. These models are divided into two parts: traditional models and modern models. Traditional models such as the waterfall model, the incremental model, the spiral model and the v-shaped model. The modern models like the agile model and extreme programming model. Where they are used in a wide range of projects in obtaining the highest proportion of productivity with minimal resources. At the end of this article is useful in a project to select one model and work it with a prototype model. Because the prototype is part of every model in the project. (Sharma, M.Kumar, 2017)

The title of this study SDLC Prototype model, advantage and disadvantage. The prototype model is an initial model of the software development life cycle. Used for the application of software under development. Which determines the product's functional behaviour. Where it differs from the original product which adheres to the exact logic. In other words, the prototype model is used to understand the client's requirements in the system. Through the prototype. The Prototype design stages consist of: collection and analysis of requirements, rapid design, modelling, customer evaluation, product refining, product engineering. Advantages of the Prototype model are design interface of the system is developed and high quality for the customer, access solutions faster through the use of feedback on initial prototypes, reduce the time and cost of the project and discover risks and errors in system design easily. The disadvantages of having a large amount of data complicate the system further, dangerous in analyze requirements because of reliance on the prototype and Reuse the developers' initial product even if it is not possible. In the end, the advantages of the model more than its disadvantages. This information was useful for the project. The prototype is suitable for use in the project as a sub-model. Because errors are quickly found and resolved through models, high efficiency to design the interface and the system with high quality and get customer feedback and improve application feedback. (Verma, 2015)

Project management is intended to manage all aspects of a project. Through the organization of all its functions and needs. There are tools used to manage projects. These tools are used to organize the stages, time of the project and to avoid the expected risk. It is useful for these tools to conduct a comprehensive study on all project tasks. This article about MS. Project tools. Is a tool used in project management. It is used to facilitate the identification of task sequences from the beginning of the project to its end and delivery. Many people use it to manage the project and reduce risk. From Microsoft, it is easy to use and learn a tool that differs from other tools. It is one of the most widely used tools nowadays. Used by projects managers and team members to complete tasks and write reports. The latest version of the tool contains many features that help them to manage and arrange tasks. Such as its new advantage in teaching new trainees to develop an e-learning project. Making it easier for everyone and increases the experience of new staff. At the end of this article about MS. Project is useful for the project. Which helps to arrange tasks and identify the sequence of tasks to and from delivery to the client. It is used in the project because of it, it is possible to create an integrated plan about the time period for each task, arranging and sequencing tasks in the project and linking each task with other tasks. In addition to the possibility of adding sources and their cost. (DeArruda, Sohmen, 2016)

\section{Analysis}




\section{Journal of Student Research}

\section{?Have you ever used this type of application before}

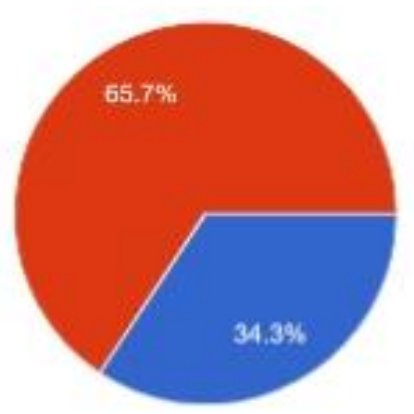

Figure 1. Data Analysis Q1

In the first question the percentage to people who have previously used the same these applications $34.3 \%$. And the proportion of people who not used same these applications $66.7 \%$. This means that these applications are not known everyone in Oman. They are not known to most people in Oman. And this type of App is new not known to most people in Oman.

\section{Will this application increase the culture of society around the laws of ?traffic safety}

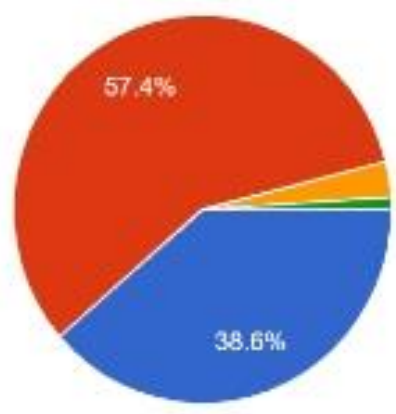

Strongly Agree' : $:$ ty

Agreel

Disagreel on

Strongly disagreei 2 نش

Figure 2. Data Analysis Q2

The second question is the percentage of people who strongly agree that this application will increase awareness about traffic safety by $39.6 \%$. The percentage of people who agreed that this application will increase awareness about traffic safety by $56.4 \%$. And $1 \%$ disagree that the application increase awareness about traffic safety. And also 3\% strongly disagree that the application increase awareness about traffic safety. This means that most people support such applications to increase awareness about traffic safety. 


\section{?Will this application increase the awareness of traffic safety}

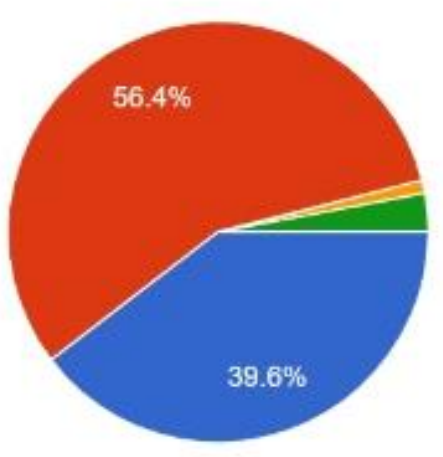

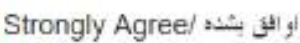

Agree/

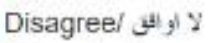

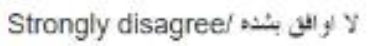

Figure 3. Data Analysis Q3

The third question is whether this application will increase the community's culture about traffic safety. Percentage of people who agree strongly 38.2\%. Percentage of the people agree that the application increases the community's culture about traffic safety are 57.8\%. And the percentage of people who do not agree that the application increases the community's culture of traffic safety is $2.9 \%$. The percentage of people who do not strongly agree is $1 \%$. This means that most people support that the application increases the community's richness about the laws in traffic safety.

\section{Figure 4. Data Analysis Q4}

The fourth question is the proportion of people who strongly agree that this type of application is useful for increasing awareness are $34.3 \%$. And the proportion of people who agree that this type of application is useful for increasing awareness are $58.8 \%$. The proportion of people who disagree that this type of application is useful for increasing awareness are $5.9 \%$. The proportion of people who strongly disagree are $1 \%$. This means that these types of applications are useful for the community and raise people's awareness.

Figure 5. Data Analysis Q5

Percentage of people who strongly agree to apply this application in Oman is $42.2 \%$. And the percentage of people who agree to apply this application in Oman is 52.9\%. Percentage of people who disagree to apply this application in Oman is $2.9 \%$. And the percentage of people who strongly disagree is $2 \%$. This means that most people support the idea of applying this application in Oman.

In the third question, which includes people's suggestions and comments about the application, its creation and its thought. Most people had positive opinions about this type of applications are important and useful to society. In increasing the awareness of people about the laws of traffic safety. And it is useful for people before entering the traditional driving education tests. Allowing them to increase and develop their knowledge about laws to avoid accidents and irregularities. And allowing them to learn before practising driving. 


\section{Conclusion}

At the end of the project and during the research and analysis of the data and project requirements. Of the sources, devices, programs and risks that are likely to occur in the project. And after conducting studies on these subjects related to the application. To analyze and know what the customer needs and what is useful to him. In addition to the feedback, people react around these applications and are useful in increasing people's culture.

\section{Future Works}

Mobile application and the application for decreasing mortality and improving the fatalities in Oman can not only increase the awareness among the young drivers but also, helps in recusing carbon emission making the environment greener, sustainable and smart (Zameer et al 2019), (Hasan et al 2016, (Mohammed 2019).

\section{Acknowledgement}

Authors would like to express their thanks to God almighty for abundant blessings because of which this research has taken its shape. Sincere thanks to the family members who have always supported while carrying out the tasks. Finally from the bottom of the heart, the authors would thank the management, staff, and friends at the Middle East College.

\section{References}

Traffic.gov, 2018. who are we. [Online] Available at: http://traffic.gov.om/?page_id=2398 [Accessed 24 2019].

SÜDOR, S., 2019. The Advantages of 3D Modelling Knowledge in Learning Unity Software. Ankara Üniversitesi Sosyal Bilimler Dergisi, 10(2), pp. 126-134.

Buyuksalih, Ismail; Bayburt, Serdar; Buyuksalih, Gurcan; Baskaraca, A. P.; Karim, Hairi; Rahman, Alias Abdul, 2017. 3D MODELLING AND VISUALIZATION BASED ON THE UNITY GAME ENGINE -- ADVANTAGES AND CHALLENGES. ISPRS Annals of Photogrammetry, Remote Sensing \& Spatial Information Sciences, 4(4), pp. 161-166

Mohov, Sergey, 2013. Practical Game Design with Unity and Playmaker : Leverage the Power of Unity 3D and Playmaker to Develop a Game From Scratch. Birmingham, UK: Packt Publishing.

Al Mazruii, Mudhar, Edwards, Jason, Armstrong, Kerry, Davey, Jeremy, 2015. Policing of road safety in Oman: Perceptions and beliefs of traffic police officers, Oman: s.n.

Sharma, Mohit Kumar, 2017. A study of SDLC to develop well-engineered software. International Journal of Advanced Research in Computer Science, 8(3), pp. 520-523.

Verma, E. P., 2015. SDLC Prototype model : Design, advantages, disadvantages and applications. [Online]

Available at: https://er.yuvayana.org/sdlc-prototype-model-design-advantages-disadvantages-andapplications/ [Accessed 45 2019].

DeArruda Marcos, Sohmen Victor, 2016. Developing eTutorials for a Core E-Tool for Project Management: MS Project 2016. Journal of Information Technology \& Economic Development, 7(2), pp. 49-64. 


\section{Journal of Student Research}

Fourth Middle East College Student Research Conference, Muscat, Sultanate of Oman

Stackify, 2019. What is SDLC? Understand the Software Development Life Cycle. [Online] Available at: https://stackify.com/what-is-sdlc/ [Accessed 16 2019].

Damedevelopertips, 2019. Spiral Model For Game Development: Techniques To Develop Games. [Online] Available at: https://gamedevelopertips.com/spiral-model-for-game-development/ [Accessed 16 2019].

Zameer, A., Saqib, M., Naidu, V.R., and Ahmed, I. (2019) 'IoT and Big Data for Decreasing Mortality Rate in Accidents and Critical Illnesses'. in 2019 4th MEC International Conference on Big Data and Smart City, ICBDSC 2019. held 2019

Hasan, R., Bhatti, A.H., Hayat, M.S., Gebreyohannes, H.M., Ali, S.I., and Syed, A.J. (2016) 'Smart Peer Car Pooling System'. in 2016 3rd MEC International Conference on Big Data and Smart City, ICBDSC 2016. held 2016

Mohammed, Q.A., Naidu, V.R., Hasan, R., Mustafa, M., and Jesrani, K.A. (2019) 'Digital Education Using Free and Open Source Tools to Enhance Collaborative Learning'. IJAEDU- International EJournal of Advances in Education 